\title{
Shakedown strength based parameter optimization technique and its application on designing an airtight module
}

\author{
Songhua Huang ${ }^{1}$, Yugong $\mathrm{Xu}^{1}$, Lele Zhang ${ }^{1}$, Geng Chen ${ }^{1 *}$, Fuming Zeng ${ }^{2}$ and Feng Liu $^{2}$
}

\begin{abstract}
Structural optimal design is traditionally performed according to the elastic limit rule which makes the structure overweighted or strength over conservative to an extent. Shakedown theory is implemented in the present study in order to measure load-capacity performance of structure. It can determine the strength of a structure under arbitrary varying loads without loading history where failure form of incremental collapse, ratcheting and alternate plasticity will be avoided. The current research subsequently provides a parameter optimal design scheme with respect to maximum structure strength and strength-to-weight performance in the framework of shakedown which makes the optimal structure design in a relatively practical way. With this motivation, the formula of proposed shakedown limited parameter optimization problem is derived and solved based on genetic-gradient coupling algorithm. The present coupling algorithm is essentially solve a double-loop problem where the inner loop adopt interior point method to solve shakedown problem under a given parameter combination while the outer loop use genetic algorithm to find the optimal parameters based on a given shakedown fitness. In addition, external database technique is applied to accelerate computation and prevent unexpected interrupt. Subsequently, a test example which is a optimal parameter design problem of plate with a circular hole is presented to demonstrate the accuracy and effectiveness of the proposed method and algorithm. In addition, the proposed method is also utilized to determine the optimal load-bearing capacity of a airtight module to be used in a manned spacecraft. Moreover, optimal shakedown load design results of the manned airtight module as well as optimal strength-to-weight efficiency design results are given in the end This study confirmed that genetic-gradient coupling algorithm is a effective means for determine the optimal parameter in accordance to the shakedown load domain.
\end{abstract}

Keywords: Parameter optimal design; Shakedown analysis; Direct Method (DM); Genetic algorithm

\section{Introduction}

The shakedown theory is known as the fundamental breakthrough of strength design and safety assessment codes. According to Melan's theorem, a maximum loading factor can be directly obtained without following the entire load history so that the structure under the shakedown loading bound will not fail due to alternating plasticity, ratcheting and instantaneous plastic collapse. For this reason, the method is also named as direct method (DM) [1, 2, 3]. The plastic limit and shakedown limit, where the former can be regarded as a particular case of the latter, have become two of the most significant reference factors

\footnotetext{
${ }^{*}$ Correspondence: gengchen@bjtu.edu.cn

${ }^{1}$ School of Mechanical, Electronic and Control Engineering, Beijing Jiaotong University, Beijing, P.R. China
}

Full list of author information is available at the end of the article for plastic failure criteria in the structure strength design under monotonic loading or cyclic loading. On the other hand, complex geometries can lead to a complicated stress distribution. When the retained local stress higher than the yield stress during the structural design, geometry parameters need to be treated with great concern to fulfill the stress constraint. However, local stress exceeds the yield stress does not mean the structural loading capacity exceeds the limit. Moreover, parameter redesign in the concept of elastic limit may lead to an unacceptable structure weight. With the concept of shakedown in structural optimization design, the full potential of a material can be utilized, and an optimal strength-to-weight parameter would be obtained simultaneously. DM leads to a massive nonlinear convex optimization problem with many unknowns. Substantial evidence suggests 
that prohibitive computing expense required to solve the problem $[4,5,6]$. That is the reason why Melan's theorem has been proven almost for a century, it is still seldom implemented as a common analysis during the structural design. Early techniques such as Simplex attempted to solve the problem by decomposing the system into a linear optimization problem [7]. In 1984, the interior-point method (IPMs) was developed, solving the large-scale nonlinear programming problem generated by DM became reasonable $[8,9,10]$. One advantage of the interior-point method, compared to active set methods such as sequential quadratic programming, is that it avoids the problem of selecting active constraints. Moreover, the number of iterations required by the interior-point method to converge is much smaller $[11,12]$. Subsequently, several optimization software packages based on the interiorpoint method were developed, such as CPLEX, LOQO, Mosek, IPOPT, and Gurobi. Some of these packages have been successfully adopted to solve DM problems. Despite this, little progress has been made in the shakedown analysis of largescale engineering structure $[13,14,15,16,17]$.

Limited by the massive optimization problem scale and low computing efficiency, the shakedown analysis is rarely used in the engineering structural optimization design, most of the researches remain in the stage of parameter sensitivity analysis. A revised sequential quadratic programming algorithm and an activeconstraint set strategy are used in Yang's research [18] to overcome the numerical difficulties while solving the limit and static shakedown problem. The effect of various shapes and sizes of typical part-through slots on the limit and shakedown loads of straight pipelines and pipe elbows are investigated and evaluated. A series of computational curves for limit and shakedown loads is obtained, and the failure modes under different loads and defects are analyzed. H. Chen et al. [19] investigated the differential impact of the ratios of bend radius to pipe mean radius $(R / r)$ and mean radius to wall thickness $(r / t)$ on limit and shakedown load envelopes of $90^{\circ}$ pipe bends under two load cases. In the subsequent research, Nak-Kyun Cho and H. Chen [20] change the research object to a $90^{\circ}$ back-to-back pipe bend structure, and use the Linear Matching Method (LMM) to create the limit, shakedown, and ratchet boundaries. Parameter sensitivity analysis finds out that $R / r$ and $r / t$ in a different type of pipe structure have consisten$\mathrm{t}$ results with the previous research. In the same way, H. Peng et al. [21] carry out the shakedown and limit analysis of $45^{\circ}$ piping elbows for varying geometry subjected to steady internal pressure and cyclic in-plane closing, opening and reverse bending moments. After various shakedown limit and plastic limit load curves are obtained, they point out that the elbows geometry parameters need to be carefully designed to create a sufficient margin between the two curves so that it can avoid unexpected plastic collapse under cyclic loads. X. Zhao [22] uses a direct cycle method to study the shakedown limit loads of perforated thick-walled cylinders under cyclic thermo-mechanical loadings. Results reveal that the shakedown limit loads decrease when the hole radius becomes larger and the wall becomes thinner. Moreover, the phenomena will be more obvious when the structure is in an axial compressive state. Y. Sun [23] introduces a numerical method to evaluate the ultimate bearing capacity of a semisubmersible brace considering cyclic wave load. The influence of load angle, shell thickness, stiffener thickness and stiffener spacing on shakedown limit were studied. Numerical results show that shakedown limit increases with the increase of shell thickness and stiffener thickness, while decreases with the increase of stiffener spacing. Kammoun and Smaoui [24, 25] utilize the plastic limit and shakedown theory to formulate statically and plastically admissible constraints. In their works, the objective is to minimize overall mass while maintaining static equilibrium and satisfying yield criterion simultaneously.

Within aforementioned works, several studies have attempted to link shakedown limit with design parameters and establish a qualitative relationship between them. However, our previous research [17, 26, 27] showed that oftentimes the relationship between load domain and design parameters is severely nonlinear and not feasible, especially for a complex engineering problem. Moreover, the relationship may extreme unsmooth and difficult to define in terms of gradients in most cases. In this type of situation, creating a numerical method for determining the optimal parameters in accordance to the shakedown load domain seems to be the best solution. With this motivation in mind, this work aimed to address a gradient-free method for parameter optimal design in terms of shakedown analysis based on DM. Genetic algorithm (GA) differ from traditional gradient based optimization methods in several significant points. The GA does not need derivative and other auxiliary information of the objective function which offer a practical way to deal with the nonlinearity in this study. GA search parallel from a population of points and therefore is easy to discover global optimum. In addition, it quite suitable for a large scale of optimization. In this study, as we only interested in the application of GA, more detailed information of GA theory and its development can be found in literatures [28, 29, 30, 31].

The remaining part of this paper proceeds as follows. Followed by the introduction, the shakedown the- 
ory and its numerical implementation are briefly elaborated. The efficient numerical forms tailored to the von Mises criterion is clarified. Subsequently, a shakedown constrained parameter optimization mathematical form is proposed. A reasonable approach to tackle computing efficiency based on a genetic-gradient coupling algorithm will be present in the following subsection. After validated by a benchmark example, the presented technique is utilized to determine the optimal connecting frame structure parameters to be used in a manned airtight module based on feasible elastic, shakedown and plastic limit load domain. Furthermore, the advantage of applying a direct method to parameter optimal design of a manned airtight module is clarified by comparing the results obtained from the shakedown analysis and elastic analysis.

\section{Methodology}

Before shakedown constrained parameter optimization method is proposed, the lower bound problem for numerical simulation is briefly introduced in Sect. 2.1 and Sect. 6. Then it will be employed later for constructing the parameter optimization problem.

\subsection{Low bound limit and shakedown theorem for elastic-perfectly plastic materials}

In the present study, the structure is idealized as an elastic-perfectly plastic material which obeys the von Mises yield condition and omits both geometrical nonlinearity and ductile damage. Considering a body $\mathcal{B}$ without body forces and subjected to multiple arbitrary varying loads. After $\mathcal{B}$ has suffered plastic deformation from an initial few cycles of external loading. The total stress field $\boldsymbol{\sigma}(\boldsymbol{x}, t)$ of $\boldsymbol{B}$ under the above hypothesis can be separated into two parts: a purely elastic stress $\boldsymbol{\sigma}^{E}(\boldsymbol{x}, t)$ and a residual stress $\rho(\boldsymbol{x}, t)$, that is,

$$
\boldsymbol{\sigma}(\boldsymbol{x}, t)=\boldsymbol{\sigma}^{E}(\boldsymbol{x}, t)+\rho(\boldsymbol{x}, t)
$$

where $\boldsymbol{x}$ indicates the position and $t$ indicates the time. The equilibrium conditions and boundary conditions have the following form after decomposition:

$$
\begin{array}{rll}
\text { equilibrium: } & \boldsymbol{\nabla} \cdot \boldsymbol{\sigma}^{E}=-\boldsymbol{f}_{V} & \boldsymbol{\nabla} \cdot \overline{\boldsymbol{\rho}}=\mathbf{0} \\
\text { statical B.C.: } & \boldsymbol{\sigma}^{E} \cdot \boldsymbol{n}=\boldsymbol{f}_{A} & \overline{\boldsymbol{\rho}} \cdot \boldsymbol{n}=\mathbf{0}
\end{array}
$$

Here, $\boldsymbol{\nabla}$ denotes the gradient of stress field, $\cdot$ denotes the dot production, $\Omega$ is the material body, $\Gamma_{1}$ is the static boundary, $\boldsymbol{n}$ is the surface normal, $\boldsymbol{f}_{A}$ is the surface loading and $\boldsymbol{f}_{V}$ is the body force which is zero under the hypothesis mentioned before. According to
Melan's theorem, a structure will shakedown if a timeindependent residual stress field $\overline{\boldsymbol{\rho}}(\boldsymbol{x})$ can be find and its superposition with $\boldsymbol{\sigma}^{E}$ multiplied by $\alpha(\alpha>1)$ satisfies the yield condition at any time $t$ and any point $\boldsymbol{x}$. Mathematical formulation for Melan's theorem can be written as:

$$
F\left(\alpha \boldsymbol{\sigma}^{E}(\boldsymbol{x}, t)+\overline{\boldsymbol{\rho}}(\boldsymbol{x})\right) \leq \sigma_{Y}^{2}(\boldsymbol{x}) \quad \forall \boldsymbol{x} \in \Omega, \forall t
$$

where $F$ stands for the von-Mises yield condition and $\sigma_{Y}$ is the yield strength, respectively.

For the structures in this study, in order to avoid unreasonable time and computational resource, equations (2) and (3) need to be carefully reformulated. During the reformulation, an important concept, load history $\mathcal{H}(\boldsymbol{x}, t)$, needs to be introduced. Considering the body $\mathcal{B}$ is simultaneously under $N L$ linearly independent varying loads $\hat{\boldsymbol{P}}_{n}(\boldsymbol{x}, t)$, then $\mathcal{H}(\boldsymbol{x}, t)$ can be defined as a superposition of these loads:

$$
\begin{aligned}
& \mathcal{H}(\boldsymbol{x}, t)=\sum_{n=1}^{N L} \hat{\boldsymbol{P}}_{n}(\boldsymbol{x}, t)=\sum_{n=1}^{N L} \mu_{n}(t) \hat{\boldsymbol{P}}_{0 n}(\boldsymbol{x}) \\
& \mu_{n}^{-} \leq \mu_{n} \leq \mu_{n}^{+}
\end{aligned}
$$

Here, $\hat{\boldsymbol{P}}_{0 n}(\boldsymbol{x})$ is a time independent loading basis, $\mu_{n}^{-}$ and $\mu_{n}^{+}$are the lower and upper bounds of load multiplier $\mu_{n}$, respectively. It is worth noting that the loading domain of $\mathcal{H}(\boldsymbol{x}, t)$ is a convex domain with each load vertex denoting as $P_{k} \quad k=1,2, \ldots, N V$ where $N V=2^{N L}$. $N V$ here stands for the number of load vertexes while $N L$ is the number of loads. Consequently, the total purely elastic stress $\boldsymbol{\sigma}^{E}[\mathcal{H}(\boldsymbol{x}, t)]$ can be illustrated briefly by a superposition of elastic stress due to different load patterns. And each elastic stress field can be viewed as time independent stress base multiplied by time dependent load multiplier.

$$
\begin{gathered}
\boldsymbol{\sigma}^{E}[\mathcal{H}(\boldsymbol{x}, t)]=\sum_{n=1}^{N L} \mu_{n}(t) \boldsymbol{\sigma}^{E}\left[\hat{\boldsymbol{P}}_{0 n}(\boldsymbol{x})\right] \\
\mu_{n}^{-} \leq \mu_{n} \leq \mu_{n}^{+}
\end{gathered}
$$

In terms of non-linear matrix inequality (3), Borkowski [32] and König [33] have proven that it is sufficient to only consider the vertices of the loading domain instead of the entire loading history $\mathcal{H}(\boldsymbol{x}, t)$. In other words, shakedown state would be obtained directly once the structure meet the conditions (2) and (3) at all loading domain vertices. Thereafter shakedown state would be achieved through any $\mathcal{H}(\boldsymbol{x}, t)$ inside the loading domain. This notable advantage of directly predict the load limit give shakedown analysis an alternate name, Direct Method. By using this approach, 
the load bearing capacity of a structure can be directly obtained without following the complicated loading history which is sometimes even inaccessible. In addition, it determines a maximal load-bearing capacity of a structure under arbitrary varying loads where failure form of incremental collapse and alternate plasticity will be avoided. Melan's theorem, as a consequence, can be rewritten in a load-path independent form as:

$$
\begin{array}{ll}
\underset{\overline{\boldsymbol{\rho}}, \alpha}{\operatorname{maximize}} & \alpha \\
\text { subject to } & \boldsymbol{\nabla} \cdot \overline{\boldsymbol{\rho}}=\mathbf{0} \quad \text { in } \Omega, \\
& \overline{\boldsymbol{\rho}} \cdot \boldsymbol{n}=\mathbf{0} \quad \text { on } \Gamma_{1}, \\
& F\left(\alpha \boldsymbol{\sigma}^{E}\left(\boldsymbol{\mu}_{k}\right)+\overline{\boldsymbol{\rho}}\right) \leq \sigma_{Y}^{2} \forall k \in[1, N V]
\end{array}
$$

Equation (6) indicates that only one load vertex is considered when $N V=1$ and the calculated $\alpha$ is equivalent to the plastic limit factor. Plastic limit analysis therefore serves as a a particular case of shakedown analysis. When two or four load vertexes are considered, i.e. $N V=2$ or $N V=4$, the results of (6) means shakedown analysis under proportionally varied or dis-proportionally varied loads. Eqn. (6) need to be reformulated into a numerical form and adapted computing acceleration strategies. The reformulation process can be found in our previous research $[26,27]$ or in Sect. 6: Appendix.

\subsection{Shakedown constrained parameter optimization problem and its mathematical form}

Directly determine the optimal parameters need to obtain the information of shakedown corresponding to the maximum $\boldsymbol{\alpha}$ under current body $\mathcal{B}_{n}$ where $n$ denotes the $n$th iteration of the optimization process. The problem can be regarded as a double-loop multivariant optimization problem where the outer loop can be written as,

$$
\begin{array}{ll}
\underset{\boldsymbol{r}}{\operatorname{maximize}} & \boldsymbol{\alpha}(\boldsymbol{r}) \\
\text { subject to } & \boldsymbol{r}_{\min } \leq \boldsymbol{r} \leq \boldsymbol{r}_{\max }
\end{array}
$$

where the variable parameter vector $\boldsymbol{r}$ determined structure form a various elastic stress $\boldsymbol{\sigma}^{E}\left(\boldsymbol{\mu}_{k}, \boldsymbol{r}\right)$ and residual stress $\overline{\boldsymbol{\rho}}(\boldsymbol{r})$ which affect the $\alpha$. As a consequence, the shakedown domain also varies simultaneously in geometric parameters. Eq. 7 indicates that structure optimization need to be proceed under the framework of shakedown analysis in order to remain the structure in a safe state. On the one hand, for a given $\boldsymbol{r}$, one can determine its shakedown limit state by solving Eq. 15. On the other hand, the complex and uncertain relationship between $\boldsymbol{\alpha}$ and $\boldsymbol{r}$ is difficult to establish. Moreover, shakedown analysis based on D$\mathrm{M}$ itself also leads to a optimization problem which is nonlinear and convex. To solve this complex nonlinear problem, genetic algorithm (GA) is used to find the optimal parameters, and the interior-point algorithm which is a gradient based method is employed to solve the shakedown problem simultaneously. The basic idea of the genetic-gradient coupling algorithm is to transform the massive integral optimization problem into a double-loop optimization problem, where the outer is a multi-variant range constraint optimization problem and the inner is a maximization optimization problem, respectively. This process will undoubtedly lead to huge computational cost. In order to improve the efficiency and further enhance the engineering practicability, external database technique is applied to avoid unnecessary repeated calculations.

\section{The implementation of genetic-gradient coupling algorithm for optimization design}

The flowchart of the proposed genetic-gradient coupling algorithm for the optimization problem described in Eq. (7) is shown in Fig. 1, and its procedure is outlined in the following steps.

Step 1. Initialize: Create a random initial population of size 50 at its initial range and write the current states of random stream to database. This information is necessary for the next run to reproduce the last results where the process may interrupt by computer power-off or unexpectable temporary failure. Storing the pseudorandom number stream at the first step instead of the end provides the advantage of not having to run the whole algorithm successfully at least one-time. In addition, the elite, crossover and mutation probability random stream generated in the following Step 4. is also recored, which is also needed in reproduction.

Step 2. Fitness calculation: Evaluate each generation of population and score each individual by the following steps:

a. Judge whether there exist the same individual, i.e. the same geometry parameter in the database. If YES, then go to step b., otherwise, go to step c. and d..

b. Read the stored fitness value from external database.

c. Begin to construct Eq. (15): Using the parameterization modeling technology to automatic generate the model geometry. Then using the Abaqus Python script to automatic generate FEM model corresponding to a unit elastic loading. The associated $\boldsymbol{\sigma}^{E}$ 
and other essential information will be obtained after submit to Abaqus. By tailoring to von Mises criterion and reformulation, the shakedown problem Eq. (15) can be handled by a commercial optimization solver such as Gurobi.

d. The new individual and shakedown load factor $\alpha$ under the current geometry set are written to the database and sent back to the main algorithm process simultaneously.

Step 3. Judge the stop conditions: The algorithm stops when the average relative change in the fitness function value over MaxStallGenerations is less than the tolerance which set as $1 \times 10^{-6}$. The MaxStallGenerations is set as 50 to ensure the problem achieve global optimal result.

Step 4. Create the next population: Individuals with the best fitness values in the current generation which called the elite are automatically survive to the next generation. Besides elite children, the algorithm creates crossover children and mutation children. Because we are interested in this paper only with the application of the genetic algorithm, the theory as well as technical details for realizing a general purposed genetic algorithm code are far beyond the scope of the study. The creation of next population in this work is based on an established MATLAB toolbox Genetic Algorithm Toolbox.

Step 5. Population re-evaluation: Re-evaluate the fitness of the new population. Go to Step 2., Step 3. and Step 4. until termination condition met. The individual in the last population with best fitness can be regarded as the optimum solution. Under this configuration, the shakedown load factor and its associated self-equilibrated residual stress would be obtained.

In addtion, the computer configuration for this study is: Dell Precision Tower 5810 Workstation, its processor is Intel $\left(\right.$ Xeon ${ }^{\circledR}$ CPU E5-1603 v3 $2.8 \mathrm{GHz}$, the operating system is Windows 10 Pro for Workstations (x64) with 128GB (DDR4 ECC memory) RAM, Matlab version is MATLABß R2015b, and Gurobi Optimizer version is 7.0.1.

\section{Test example of the proposed work flow}

The problem of a plate with a circular central hole subjected to surface tractions perpendicular to the edges of the plate is a classical example for shakedown analysis. Our previous research [26, 27] confirmed the plastic limit and shakedown results of this example are in high agreement with the literatures, which further demonstrates our technique for shakedown analysis to be a viable and suitable numerical approach. Therefore, this benchmark problem is also employed in this section to illustrate the accuracy, reliability and efficiency of the proposed work flow in optimal designing structure in terms of shakedown load domain.

The length of the plate $L_{1}$ and $L_{2}$, shown in Fig. 2a, are set as design parameter a and parameter $\mathrm{b}$ which both vary from $22 \mathrm{~mm}$ to $100 \mathrm{~mm}$, other parameters are set as constant where diameter $D=20 \mathrm{~mm}$ and thickness $h=2 \mathrm{~mm}$. The material properties of the model are shown in Table 1 . Only $1 / 4$ of the geometry is submitted to the work flow thanks to the symmetry of the model. Moreover, for simplicity, only $P_{x}$ is taken into account. The settings of GA used in this example can be summarized as follows. The maximum number of generation and stall generations are set to 200 and 50 , respectively. The elite count is $5 \%$ of the population size, rounded up. Crossover fraction is $80 \%$ and uniform mutation rate is set as $10 \%$, respectively. The starting individual set randomly of the range.

Before giving the results of GA, the optimal result for this example, $L_{1}=50 \mathrm{~mm}, L_{2}=50 \mathrm{~mm}$, can be estimated by $10 \times 10$ uniform sampling calculation which can be seen from Fig. 4. Under the ideal configuration, the purely elastic stress field is shown in Fig. 2b, and its shakedown result is 1.79 . Fig. 3 shows the iteration process of the plate with a circular central hole subjected to surface tractions $P_{x}$. The total generation is 135, and there are 50 individuals of each generation submit to the process. As a result of fact, there are 6750 iterations in total. From the graph we can see that the two design parameters are converge to a stable state where $L_{1}=50.00 \mathrm{~mm}, L_{2}=43.50 \mathrm{~mm}$. In Fig. 3, mean fitness value increase steadily during the first half of the generations and then reach to an maximum level until the end of the iteration. In the end, optimization process quit after 6750 iterations due to average change in the fitness value less than $10^{-6}$. Under the obtained optimal results, the shakedown factor is 1.78 which means the plate would not fail due to instantaneous plastic collapse, ratcheting or alternating plasticity when $P_{x} \leq 178.30 \mathrm{MPa}$.

The relative error between the obtained results and the expectation is $0.50 \%$ which is within acceptable accuracy. Nevertheless, a possible explanation for the small relative error may be the lack of adequate searching space and a proper initial starting guess point of GA. For a better understanding, $10 \times 10$ uniform sampling calculation for fitting shakedown factor surface is shown in Fig. 4. The fitting surface is quite smooth which gives another gradient based optimization method to validate the accuracy of the outer loop. With this motivation, optimization algorithm of the outer loop described in Fig. 1 is tested with IPMs to illustrate the acceptable accuracy of GA. Fig. 4 shows the searching points between GA and IPMs. Table. 2 
compares the results obtained by the two optimization method. Relative errors and computing time are also presented in the table.

Fig. 4 and Table. 2 indicate that the optimization process of GA is a gradual step-by-step process while IPMs is not, and the optimal results can be quickly obtained by IPMs. This could be attributed to the smoothness feature of target surface. Gradient based algorithm like IPMs is quite suitable for solving this type of problem while gradient-free algorithm like GA can also obtain the optimal solution within acceptable accuracy. It is also indicate that the total iteration step of GA is less than IPMs's, whereas computing time is not. This could be attributed to the different actual computing steps. Although the computing step of GA is 2600 due to each generation of GA include 50 individuals, the actual computing total step is 1094 after judging the duplicated individuals (Step Step 2. a. in Sect. 3). While the actual computing total step of IPMs remains 290 steps and would not decrease due to the algorithm itself is based on gradient descent principle and will not compute repeatedly. As a consequence of fact, the different of the computing time is make sense.

\section{Optimization design of a manned airtight module}

\subsection{Description of the problem}

In Fig. 5a, international space station and a typical manned spacecraft are shown in figures as examples [34] to illustrate the position of a manned airtight module. The module generally consists of wall panels, connected frames, scuttles and other components where the wall panels and connected frames contribute most of the weight. The connected frame structure , which can be seen from Fig. 5b, is not only used to support the entire structure but also designed for realizing some additional functions or installing other equipment. As a result, the structure geometries can be quite complex and lead to a complicated stress distribution where the local stress of some area can exceed the tolerance value. Our previous research [17] investigated the parametric sensitivity in terms of the shakedown and plastic limit principle, which demonstrates that optimization is necessary to obtain an optimal result for both load capacity and lightweight design. The present section was designed to determine the optimal combination of the designed parameters and optimal strength-to-weight ratio of the manned airtight module under internal pressure $0.15 \mathrm{MPa}$. As the connecting structure is the most important part of the manned airtight module, its geometrical complexity in a given fully detailed size is shown in Fig. 5b. The present optimization design of a manned airtight module adopt the same strategy with the plate a circular hole in Sect. 4. All variables in the figure are remained the same except dimensions a and $\mathrm{b}$ whose original values are $7 \mathrm{~mm}$ and $8 \mathrm{~mm}$, respectively. The varying range of the dimensions is $5 \leq a \leq 11$ and $6 \leq b \leq 12$, the material properties of aluminum alloy used in manned airtight module are shown in Table 3. It is convenient to perform the current study based on axisymmetric elements (CAX4) on account of the symmetric structure and its symmetric boundary conditions. The airtight FE model is auto-meshed with a varying range of 45,353 46,456 linear quadrilateral elements and 51,224 52,319 nodes during optimization process.

\subsection{Results and discussion}

Before present the optimization results and in order to better understand the objective surfaces of the optimization, $13 \times 13$ uniform sampling calculation for fitting surfaces of plastic limit, shakedown and elastic limit factors is adopted. Fig. 6 shows the relationship between three load limits domain and the selected parameters. The figure 6 is quite revealing in several ways. First, the shakedown and elastic load surface is quite uneven and has lots of local peaks and valleys which is quite suitable for GA optimization instead of gradient based optimization method like IPMs. In addition, the uneven load surface, on the other hand, demonstrates that commonly used surrogate model method is not suitable for optimization in terms of shakedown theory, especially for a large engineering problem. Otherwise, high amount of shakedown details would miss. Second, the elastic limit surface is far below the plastic limit and shakedown load surface which means evaluating the load capacity performance based on elastic limit principal might not fully utilize abundant material potential, particularly for the manned airtight module structure under long-life demand during flight. Third, when $a<8, b<7.5$, increasing the parameter $\mathrm{a}$ and $\mathrm{b}$ will lead to an enhanced structure strength. When $a>8, b>7.5$, although the plastic limit increases and saturates, the shakedown limit may even decrease and the surface become uneven.

The population distribution of selected generations is presented in the Fig. 7. There is a clear trend that the distribution is gradually become narrow. 50 individuals almost overlap after generation 20. Individuals with the best fitness values are survive to the final generation. Table 4 presents the results obtained from the preliminary analysis of the two-parameter optimization with respect to maximize shakedown factor. For a better comparison, the table also show the optimization results when object changes to maximize 
elastic limit factor and plastic limit factor. As the mass of module also play an important role and to assess strength-to-weight performance of manned airtight module structure, a efficiency factor is defined and notated as $\eta=\frac{\alpha_{S D}}{m}$ where $m$ is the total mass of airtight module under current configuration in the iteration step. And the problem 7 turn into maximize this defined ratio $\eta$. Fig. 8 present the convergence process of $\eta$. The results show that the total generation is 52 and the gap between maximum fitness and minimum fitness narrowed rapidly before generation 12 . Gap between the two curves narrowed to almost zero after generation 34. The global optimal individual first appears also at generation 34 and the curves flatted after the generation 18. Curves are set axis breaks at generation 35 and generation 50 due to slightly changes. Table 4 also presents the optimal $\eta$ results when the optimization process satisfied the termination criterion.

As can be seen from the Table 4, structure under the original design parameters 7 and 8 are already meet the service requirement under the internal pressure of $0.15 \mathrm{MPa}(\alpha=1)$. However, whether to achieve a greater elastic limit or a greater shakedown limit, the two parameters would be adjust to increase parameter $\mathrm{a}$ and decrease parameter $\mathrm{b}$ simultaneously compared to the original design. On the other hand, the optimal results under plastic limit design almost reaching the upper bound of the constraint range, it seems believe that the more material is, the larger plastic limit load airtight module structure can reach. Unsurprisingly, this trend can also be seen from Fig. 6. In addition, for a greater strength-to-weight performance, the combination 10.22 and 7.22 should be taken into consideration so that an advantageous combination of load capacity and light weight can be attained simultaneously. With the employ of axisymmetric elements CAX4, calculating source demand of this airtight example is under control. Fig. 9 gives the equivalent von Mises stress distribution of the two recommended optimized manned airtight module under the internal pressure of $0.15 \mathrm{MPa}$. The most critical position of the original or optimal structure all appear at the connected structure under the frame. In contrast, the maximum displacement and maximum equivalent von Mises stress of optimal design are all lower than to the original design. However, what is noteworthy is that there are no significant relationship between von Mises stress and shakedown load result. As the optimal elastic limit design result in lowest maximum von Mises stress, but the bearing capacity is not the optimal solution.

\section{Conclusion}

The current research present a mathematical and algorithmic framework for producing optimal designs of structures exhibit maximum shakedown loading capacity, which is an innovative means to evaluate the strength of structure compared with elastic limit loading capacity. The integral problem is transformed into solving a double-loop multi-variant optimization problem. The proposed numerical optimization method is validated by a classical problem and subsequent adopted in optimal parameter design of the connecting structure to be used in a manned airtight module. The results of this study support the idea that shakedown analysis should be used in optimization design of airtight module instead of using the traditional elastic limit rule. As the load capacity evaluate by shakedown analysis would be more factual. This study confirmed that GA is a viable means for determine the optimal parameter in accordance to the shakedown load domain. In the end, the research suggest an optimal result for both load capacity and lightweight design for the manned airtight module. Whilst this study only test two design parameters and one object in the optimization, further research might investigate the potential ability of the coupling algorithm for multiparameter and multi-object optimization.

\section{Declarations}

Availability of data and materials

The datasets generated during and/or analysed during the current study are not publicly available but are available from the corresponding author on reasonable request.

Competing interests

The authors declare that they have no competing interests.

Funding

This work is supported by the National Natural Science Foundation of China (Grant No. 52075033) and Fundamental Research Funds for the Central Universities (Grant No. 2020RC202)

Authors' contributions

Songhua Huang: conceptualization; performing the simulation; preparation of data presentation and visualization; designing computer programs; writing original draft preparation

Yugong $\mathrm{Xu}$ : conceptualization; laboratory resources; provided guidance in theory

Lele Zhang: conceptualization; computing resources; project administration; writing review \& editing ; funding acquisition

Geng Chen: conceptualization; development of methodology; assisted with laboratory analyses; conducting results analysis and validation; manuscript review \& editing; funding acquisition

Fuming Zeng: conceptualization; laboratory resources

Feng Liu: conceptualization; laboratory resources

All authors discussed the results and contributed to the final manuscript

Acknowledgements

The authors gratefully acknowledge reviewers for spending time reading this work and giving insightful suggestions.

Author details

${ }^{1}$ School of Mechanical, Electronic and Control Engineering, Beijing Jiaotong University, Beijing, P.R. China. ${ }^{2}$ Beijing Institute of Spacecraft System Engineering, China Academy of Space Technology, Beijing, P.R. China. 
References

1. Chen, G., Bezold, A., Broeckmann, C., Weichert, D.: On the statistical determination of strength of random heterogeneous materials. Composite Structures 149, 220-230 (2016)

2. Hachemi, A., Chen, M., Chen, G., Weichert, D.: Limit state of structures made of heterogeneous materials. International Journal of Plasticity 63, 124-137 (2014)

3. Weichert, D., Maier, G.: Inelastic Analysis of Structures Under Variable Loads : Theory and Engineering Applications. Solid Mechanics and its Applications. Kluwer Academic Publishers, Dordrecht ; Boston (2000)

4. Staat, M., Heitzer, M., Lang, H., Wirtz, K.: Direct finite element route for design-by-analysis of pressure components. International Journal of Pressure Vessels and Piping 82(1), 61-67 (2005)

5. Akoa, F., Hachemi, A., An, M. L.T.H. Said, Tao, P.D.: Application of lower bound direct method to engineering structures. Journal of Global Optimization 37(4), 609-630 (2007)

6. Skordeli, M.-A., Bisbos, C.: Limit and shakedown analysis of $3 \mathrm{~d}$ steel frames via approximate ellipsoidal yield surfaces. Engineering Structures 32(6), 1556-1567 (2010)

7. Corradi, L., Zavelani, A.: A linear programming approach to shakedown analysis of structures. Computer Methods in Applied Mechanics and Engineering 3(1), 37-53 (1974)

8. Karadeniz, S., Ponter, A.R.S.: A linear programming upper bound approach to the shakedown limit of thin shells subjected to variable thermal loading. Journal of Strain Analysis for Engineering Design 19(4), 221-230 (1984)

9. Karmarkar, N.: A new polynomial-time algorithm for linear programming. In: Proceedings of the Sixteenth Annual ACM Symposium on Theory of Computing, pp. 302-311 (1984). ACM

10. Forsgren, A., Gill, P.E., Wright, M.H.: Interior methods for nonlinear optimization. SIAM Review 44, 525-597 (2002)

11. Nguyen, A.D.: Lower-bound shakedown analysis of pavements by using the interior point method. PhD thesis, RWTH Aachen University (2007)

12. Chen, G.: Strength prediction of particulate reinforced metal matrix composites. PhD thesis, RWTH Aachen University (2016)

13. Weichert, D., Maier, G.: Inelastic Analysis of Structures Under Variable Loads: Theory and Engineering Applications vol. 83. Springer, ??? (2001)

14. François, A., Abdelkader, H., Said, M., Tao, P.D., et al.: Application of lower bound direct method to engineering structures. Journal of Global Optimization 37(4), 609-630 (2007)

15. Simon, J.-W., Weichert, D.: Numerical lower bound shakedown analysis of engineering structures. Computer Methods in Applied Mechanics and Engineering 200(41), 2828-2839 (2011)

16. Wen, J., Gao, X., Xuan, F., Tu, S.: Autofrettage and shakedown analyses of an internally pressurized thick-walled spherical shell based on two strain gradient plasticity solutions. Acta Mechanica 228(1) 89-105 (2017)

17. Li, W., Zeng, F., Chen, G., Deng, Y., Liu, G., Zhang, X., Bezold, A., Broeckmann, C.: Shakedown analysis for structural design applied to a manned airtight module. International Journal of Pressure Vessels and Piping 162, 11-18 (2018)

18. Yang, B., Chen, G., Xu, Z., Xu, B.: Shakedown and limit analysis of defective pressure vessels. Tsinghua Science and Technology 6(4), 326-330 (2001)

19. Chen, H., Ure, J., Li, T., Chen, W., Mackenzie, D.: Shakedown and limit analysis of 90 pipe bends under internal pressure, cyclic in-plane bending and cyclic thermal loading. International journal of pressure vessels and piping 88(5-7), 213-222 (2011)

20. Cho, N.-K., Chen, H.: Shakedown, ratchet, and limit analyses of 90 back-to-back pipe bends under cyclic in-plane opening bending and steady internal pressure. European Journal of Mechanics-A/Solids 67, 231-242 (2018)

21. Peng, H., Shen, J., Liu, Y., Chen, H.: Limit and shakedown analysis of 45-degree piping elbows under internal pressure and in-plane bending (2019)

22. Zheng, X., Xuan, F.: Autofrettage and shakedown analysis of strain-hardening cylinders under thermo-mechanical loadings. The Journal of Strain Analysis for Engineering Design 46(1), 45-55 (2011)

23. Sun, Y., Shen, S.-I., Zhao, J.-I.: Influence of material properties on analysis and design of pavements using shakedown theory. Journal of Shanghai Jiaotong University (Science) 15(3), 329-333 (2010)

24. Kammoun, Z., Smaoui, H.: A direct method formulation for topology plastic design of continua. In: Direct Methods for Limit and Shakedown Analysis of Structures, pp. 47-63. Springer, ??? (2015)

25. Kammoun, Z., Fourati, M., Smaoui, H.: Direct limit analysis based topology optimization of foundations. Soils and Foundations 59(4), 1063-1072 (2019)

26. Huang, S., Xu, Y., Bezold, A., Zhang, L., Chen, G., Broeckmann, C.: A direct method-based strength evaluation of the cast aluminum beam used in a high-speed train. Proceedings of the Institution of Mechanical Engineers, Part F: Journal of Rail and Rapid Transit 233(9), 896-905 (2019)

27. Huang, S., Xu, Y., Chen, G., Zhang, L., Bezold, A., Qin, F.: A numerical shakedown analysis method for strength evaluation coupling with kinematical hardening based on two surface model. Engineering Failure Analysis 103, 275-285 (2019)

28. Konak, A., Coit, D.W., Smith, A.E.: Multi-objective optimization using genetic algorithms: A tutorial. Reliability Engineering \& System Safety 91(9), 992-1007 (2006)

29. Feng, K., Lu, Z., Ling, C., Yun, W., He, L.: An efficient computational method for estimating failure credibility by combining genetic algorithm and active learning kriging. Structural and Multidisciplinary Optimization, 1-15 (2020)

30. Deb, K., Pratap, A., Agarwal, S., Meyarivan, T.: A fast and elitist multiobjective genetic algorithm: Nsga-ii. IEEE transactions on evolutionary computation 6(2), 182-197 (2002)

31. Wang, L., Xia, X., Cao, J., Liu, X., Liu, J.: Improved ant colony-genetic algorithm for information transmission path optimization in remanufacturing service system. Chinese Journal of Mechanical Engineering 31(1), 107 (2018)

32. Borkowski, A., Kleiber, M.: On a numerical approach to shakedown analysis of structures. Computer Methods in Applied Mechanics \& Engineering 22(1), 101-119 (1980)

33. König, J.A.: Shakedown of Elastic-plastic Structures. Fundamental Studies in Engineering. Elsevier, ??? (1987)

34. NASA: Space Station (2017). https : //www.nasa.gov/mission_pages/station/images/index.html

\section{Figures}

\section{Tables}

Table 1 Material properties for steel in Sect. 4

\begin{tabular}{ccc}
\hline$E$ & $\boldsymbol{\nu}$ & $\sigma_{Y}$ \\
\hline $210 \mathrm{GPa}$ & 0.3 & $280 \mathrm{MPa}$
\end{tabular}

Table 3 Material properties used in manned airtight module of Sect. 5

\begin{tabular}{cccc}
\hline$\rho$ & $E$ & $\nu$ & $\sigma_{Y}$ \\
\hline $2.7 \mathrm{~g} / \mathrm{cm}^{3}$ & $68 \mathrm{GPa}$ & 0.3 & $130 \mathrm{MPa}$
\end{tabular}

Appendix: Formulation of the lower bound problem for numerical simulation

We consider a structure discretized into $N E$ elements with a total of $N K$ nodes and $N G$ Gaussian points. Each element has $N G K$ nodes and $N G E$ Gaussian points. Then, the following formula (8) can be derived by noticing that the virtual work contribution does not contain self-equilibrated residual stress $\bar{\rho}$ related part, i.e. $\overline{\boldsymbol{\rho}}$ is not doing any virtual work. 
Table 2 Comparison of the results obtained by GA and IPMs

\begin{tabular}{|c|c|c|c|c|c|c|}
\hline \multirow{2}{*}{-} & \multirow{2}{*}{ Generations } & \multicolumn{4}{|c|}{ Results } & \multirow{2}{*}{ Computing time $/[h]$} \\
\hline & & $L_{1} /[m m]$ & $L_{2} /[m m]$ & $\alpha /[-]$ & Relative error & \\
\hline GA & 135 & 50.00 & 43.50 & 1.78 & $0.50 \%$ & 101.28 \\
\hline IPMs & 290 & 49.99 & 48.84 & 1.79 & $0.14 \%$ & 19.28 \\
\hline Expectation & - & 50.00 & 50.00 & 1.79 & - & - \\
\hline
\end{tabular}

Table 4 Optimization results of manned airtight module and the associated load limit factors

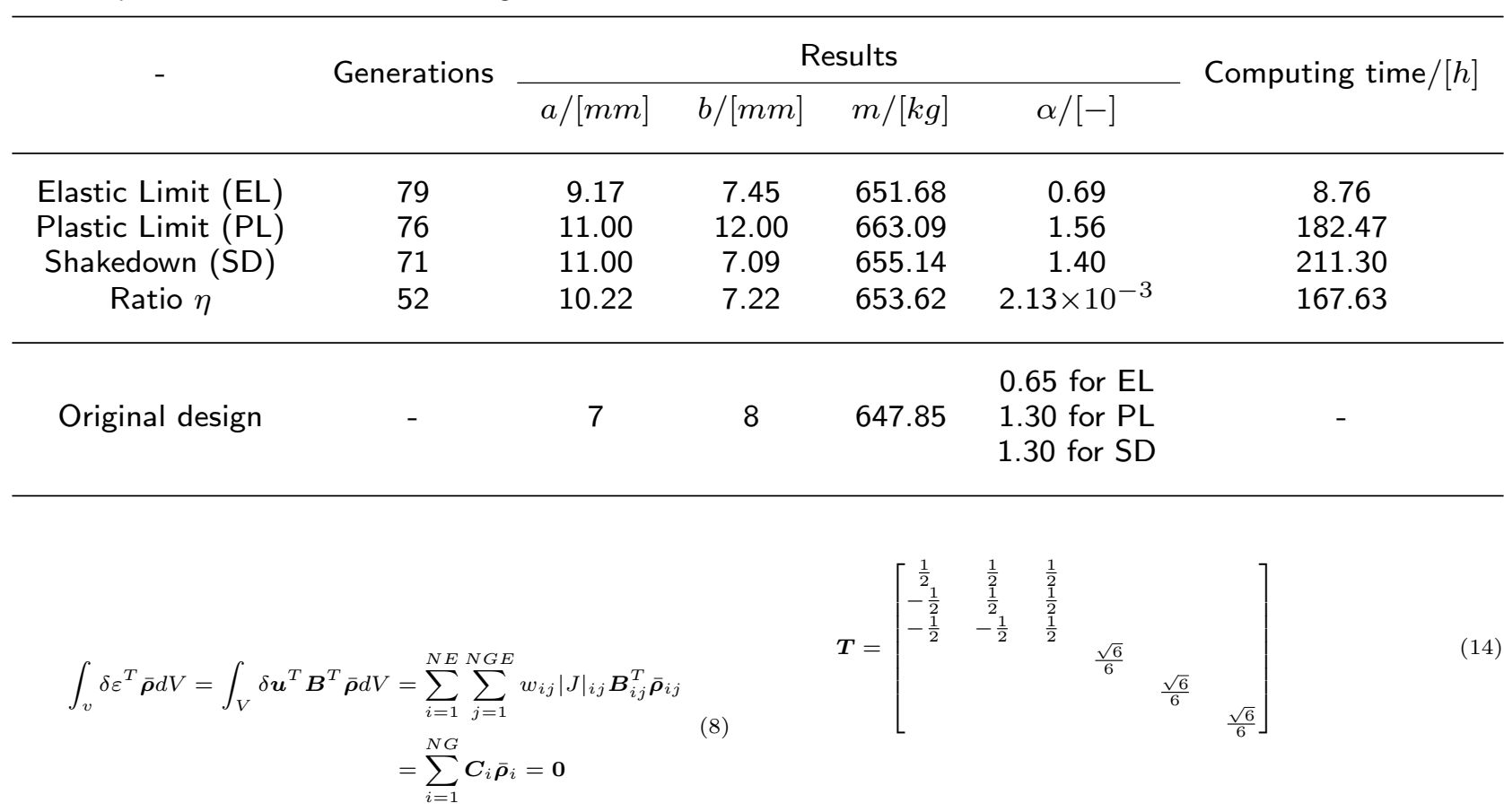

Here, $w_{i j}$ is referred to as the weight factor of Gaussian point $j$ in element $i, J$ is the Jacobian matrix, $\boldsymbol{B}$ is dubbed the strain-displacement matrix, $\delta \boldsymbol{u}$ is a variation of virtual displacement and $\boldsymbol{C}$ is a self-equilibrium matrix. Additionally, following Akoa's strategy [14] of tailoring to von Mises criterion, one can let

$$
\begin{aligned}
& \boldsymbol{M}_{r}=\sqrt{2} \sigma_{Y_{r}} \boldsymbol{D}_{r} \boldsymbol{L}^{-T} \\
& \boldsymbol{D}_{r}=\left[\begin{array}{lllll}
\left(\boldsymbol{C}_{r} \boldsymbol{T}\right)^{1} & \left(\boldsymbol{C}_{r} \boldsymbol{T}\right)^{2} & \left(\boldsymbol{C}_{r} \boldsymbol{T}\right)^{4} & \left(\boldsymbol{C}_{r} \boldsymbol{T}\right)^{5} & \left(\boldsymbol{C}_{r} \boldsymbol{T}\right)^{6}
\end{array}\right] \\
& \boldsymbol{N}=\left[\begin{array}{llll}
\left(\boldsymbol{C}_{1} \boldsymbol{T}\right)^{3} & \left(\boldsymbol{C}_{2} \boldsymbol{T}\right)^{3} & \ldots & \left(\boldsymbol{C}_{N G} \boldsymbol{T}\right)^{3}
\end{array}\right] \\
& \boldsymbol{w}_{1}=\sum_{r=1}^{N G} \boldsymbol{C}_{r} \boldsymbol{\sigma}_{r 1}^{e}
\end{aligned}
$$

where

$$
\boldsymbol{L}=\left[\begin{array}{lllll}
\sqrt{2} & & & & \\
\frac{\sqrt{2}}{2} & \frac{\sqrt{6}}{2} & & & \\
& & 1 & & \\
& & & 1 & \\
& & & & 1
\end{array}\right]
$$

then we may rephrase (6) as the following form for two load cases, i.e., $N L=2$.

$$
\begin{aligned}
& \underset{\boldsymbol{u}_{r 1}^{\prime} \boldsymbol{u}_{r 2}, \boldsymbol{y}_{1}, \alpha}{\operatorname{minimize}}-\alpha \\
& \text { subject to } \sum_{r=1}^{N G} \boldsymbol{M}_{r}\left(\boldsymbol{u}_{r 1}\right)+\boldsymbol{N}\left(\boldsymbol{y}_{1}\right)-\alpha \boldsymbol{w}_{1}=\mathbf{0}, \\
& \boldsymbol{u}_{r 1}-\boldsymbol{u}_{r 2}=\alpha \boldsymbol{\gamma}_{r}, \\
&\left\|\boldsymbol{u}_{r 1}\right\|^{2} \leq 1, \\
&\left\|\boldsymbol{u}_{r 2}\right\|^{2} \leq 1, \\
& r=1, \ldots, N G .
\end{aligned}
$$

It is worth noting that such mathematical form can be extended to the three or more load cases which is usually called high dimension shakedown form. $\|\cdot\|^{2}$ here is the Euclidean norm of a vector, $\gamma_{r}$ represents a column vector in $\mathbb{R}^{5}$ whose five components corresponding to the first five rows of $\boldsymbol{S}\left(\boldsymbol{\sigma}_{r 1}^{e}-\boldsymbol{\sigma}_{r 2}^{e}\right)$. Observe the optimization problem (6) and (15), one may draw a conclusion that the rephrased optimization problem is regular, nonlinear and convex. In addition, one may view $\boldsymbol{u}_{r 1}$ and $y_{r 1}$ as a variable 


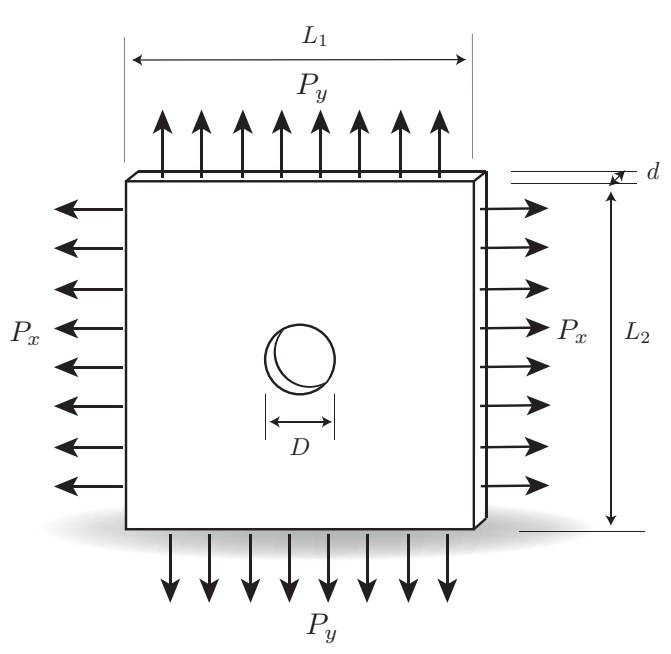

(a) Sketch of the model and its details

$$
P_{x}=100 M P a
$$

\section{S, Mises}
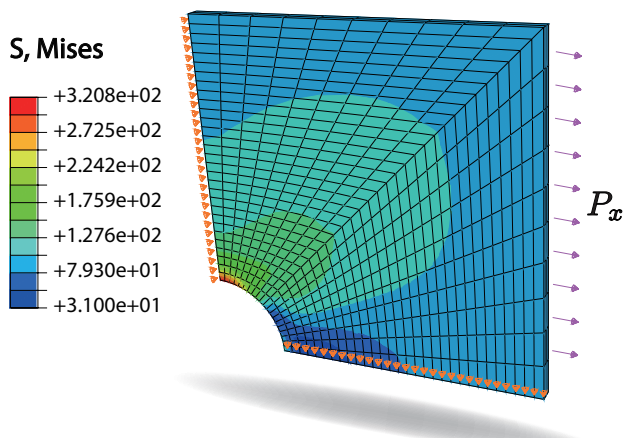

(b) Purely elastic stress fields under the load $P_{x}$ where $L_{1}=L_{2}=50 \mathrm{~mm}$

Figure 2 Sketch and elastic stress fields of plate with a circular central hole

substitution of $\boldsymbol{\sigma}_{r 1}$ :

$$
\begin{aligned}
& \left(\begin{array}{l}
\boldsymbol{u}_{r 1} \\
y_{r 1}
\end{array}\right)=\left(\begin{array}{l}
u_{r 1}^{1} \\
u_{r 1}^{2} \\
u_{r 1}^{3} \\
u_{r 1}^{4} \\
u_{r 1}^{5} \\
y_{r 1}
\end{array}\right) \\
& =\left[\begin{array}{cccccc}
\frac{1}{\sigma_{Y_{r}}} & -\frac{1}{2 \sigma_{Y_{r}}} & -\frac{1}{2 \sigma_{Y_{r}}} & & & \\
& \frac{\sqrt{3}}{2 \sigma_{Y_{r}}} & -\frac{\sqrt{3}}{2 \sigma_{Y_{r}}} & & & \\
& & & \frac{\sqrt{3}}{\sigma_{Y_{r}}} & & \\
& & & & \frac{\sqrt{3}}{\sigma_{Y_{r}}} & \\
& & & & & \frac{\sqrt{3}}{\sigma_{Y_{r}}}
\end{array}\right]\left(\begin{array}{c}
\sigma_{r 1}^{1} \\
\sigma_{r 1}^{2} \\
\sigma_{r 1}^{3} \\
\sigma_{r 1}^{4} \\
\sigma_{r 1}^{5} \\
\sigma_{r 1}^{6}
\end{array}\right)
\end{aligned}
$$

Such variable substitution also serve the tailoring strategy which will leading to a better computational efficiency. Eq. (15) is a typical second order cone programming (SOCP) problem which can be solved quickly using the interior-point methods (IPMs). The problem is then submitted to Gurobi

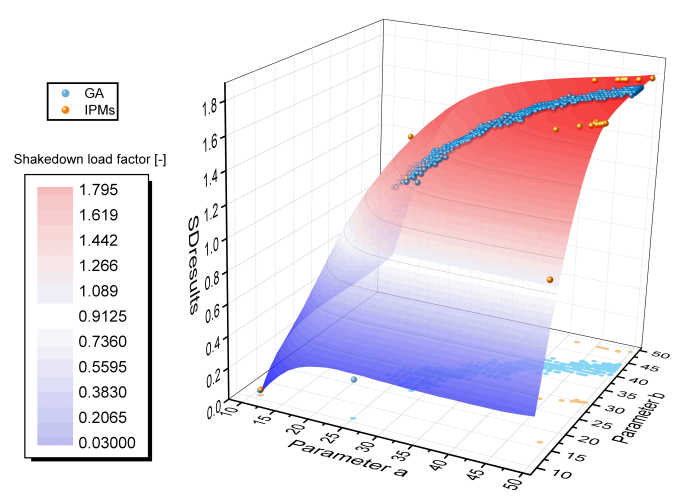

(a) Fitting surface of shakedown factors along with two parameters and optimization searching points of GA and IPMs

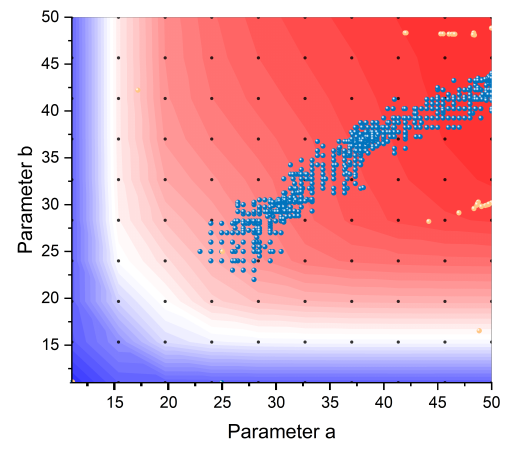

(b) Projection graph of $4 a$

Figure 4 The fitting surface of shakedown factors and its projection which is a SOCP powerful solver based on IPMs to calculate the maximum load factor $\alpha$ and its associated self-equilibrated residual stress $\overline{\boldsymbol{\rho}}$. 


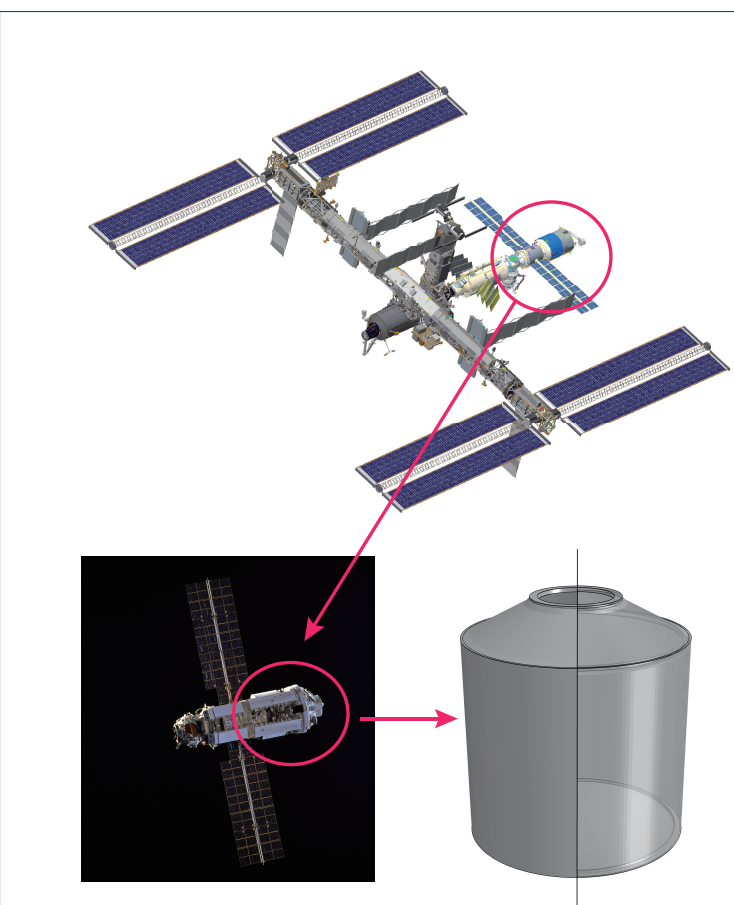

(a) A typical structure of the manned airtight module

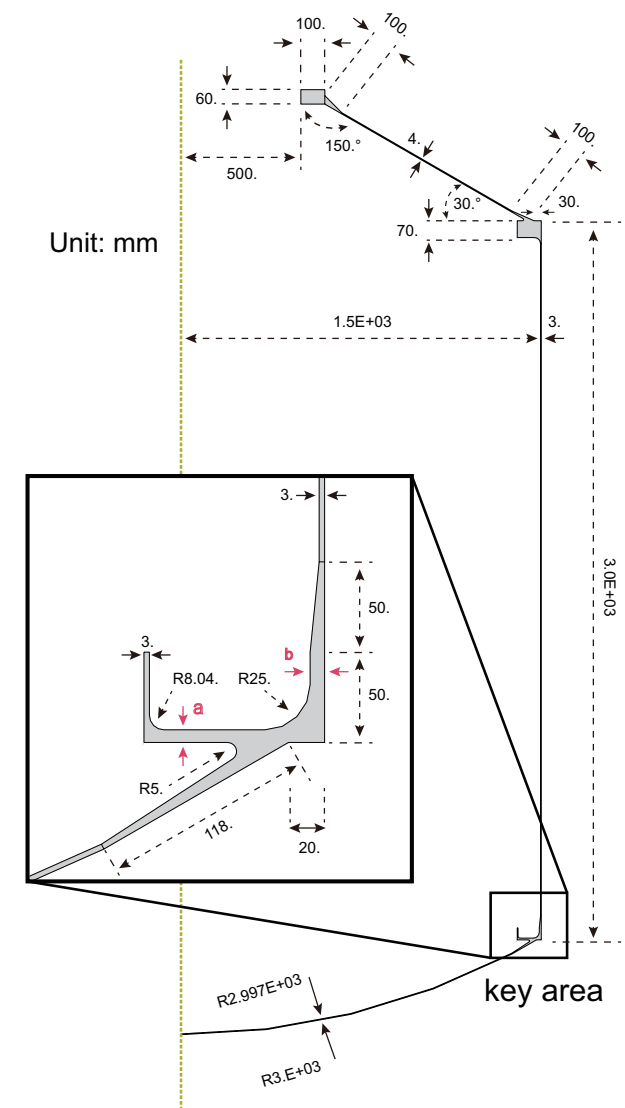

(b) Sketch of a given manned airtight module structure

Figure 5 A typical structure of the airtight module from manned spacecraft and its sketch

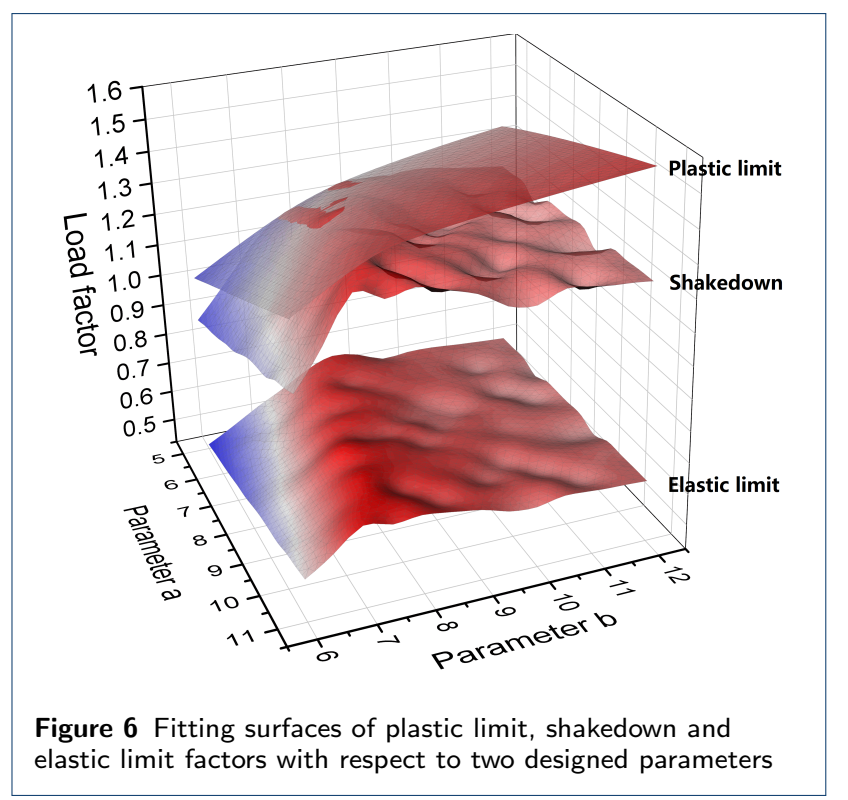




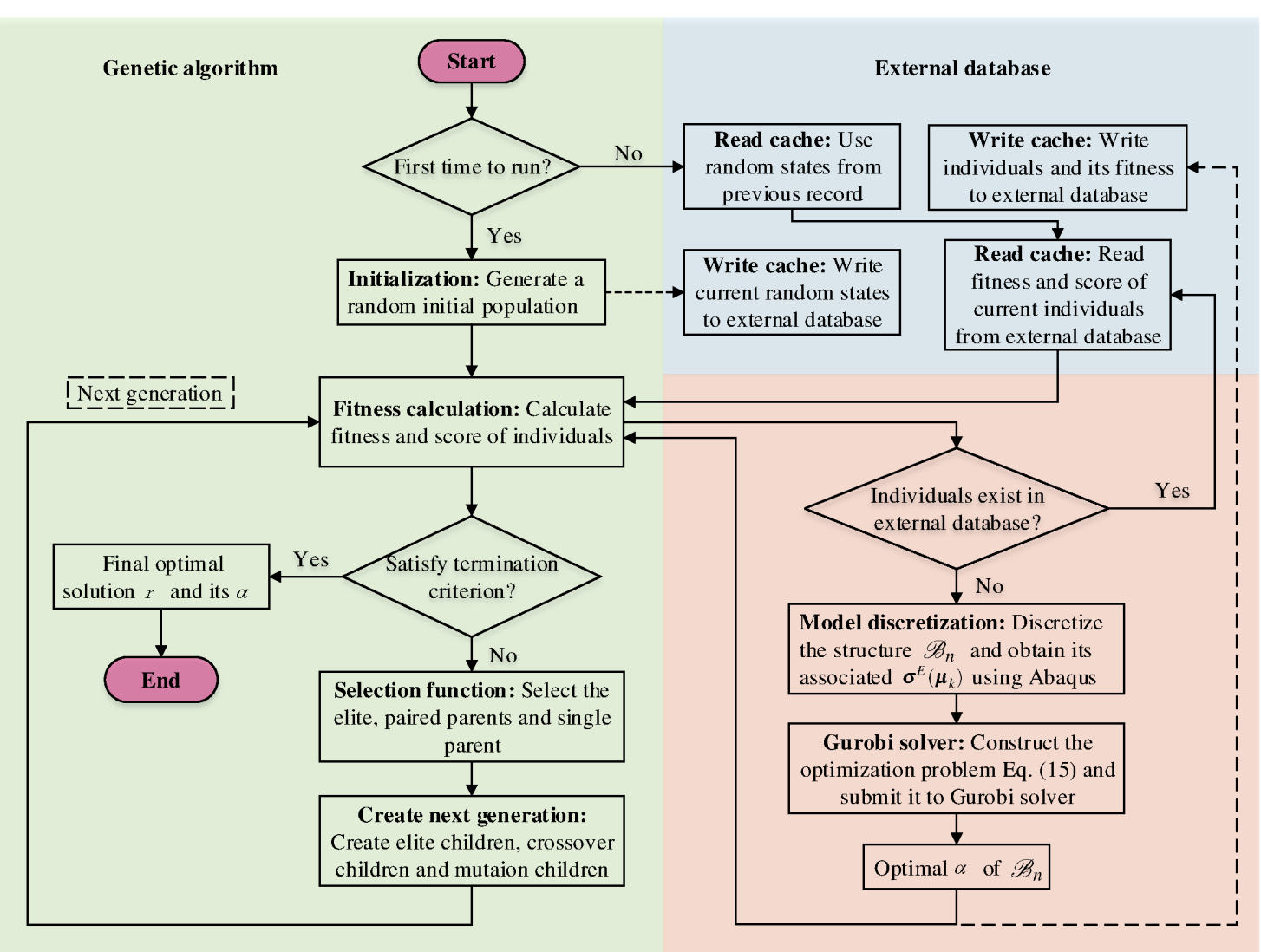

Figure 1 Flowchart of genetic-gradient coupling algorithm for structure optimization design

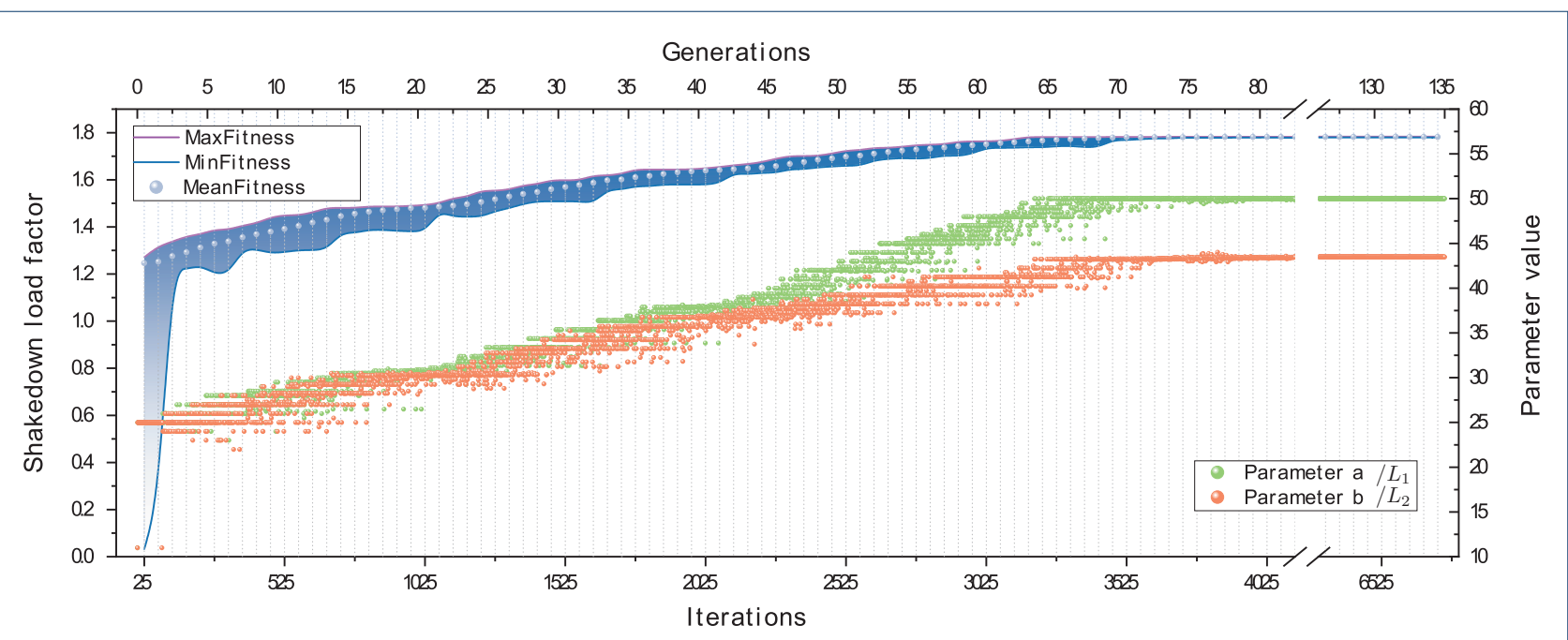

Figure 3 Convergence process of the present algorithm 


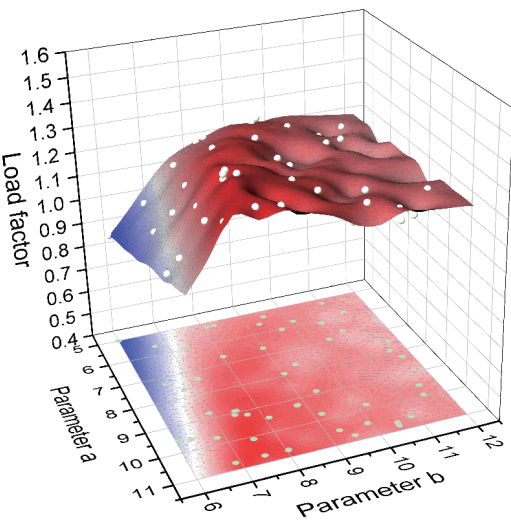

Generation 1 \& Initial generation

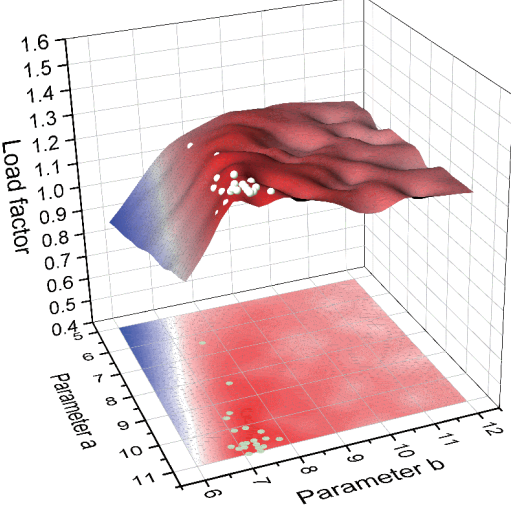

Generation 10

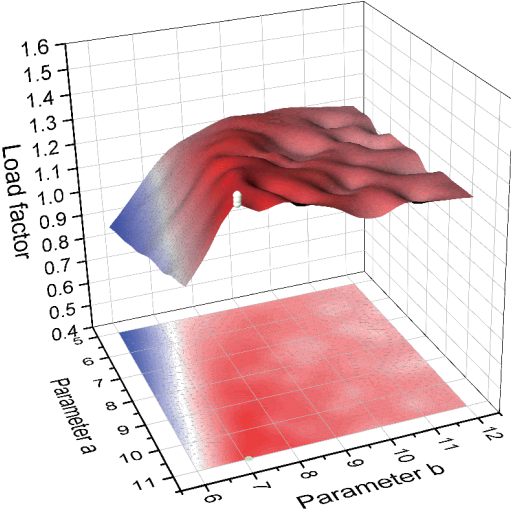

Generation 20

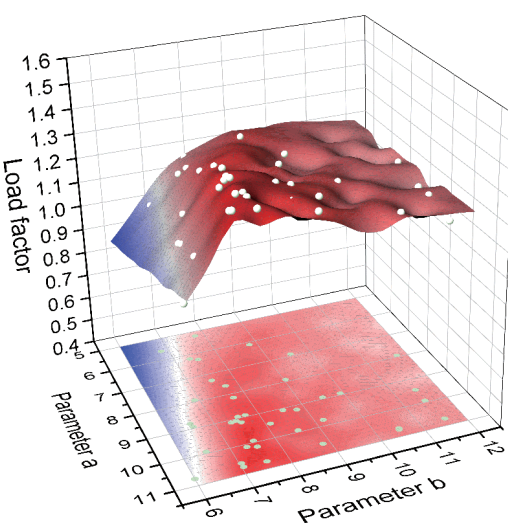

Generation 3

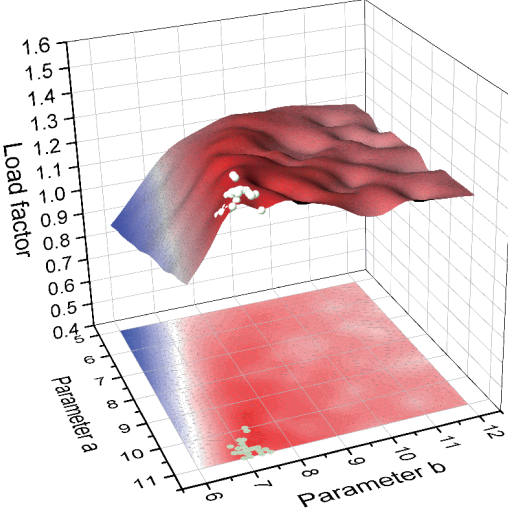

Generation 12

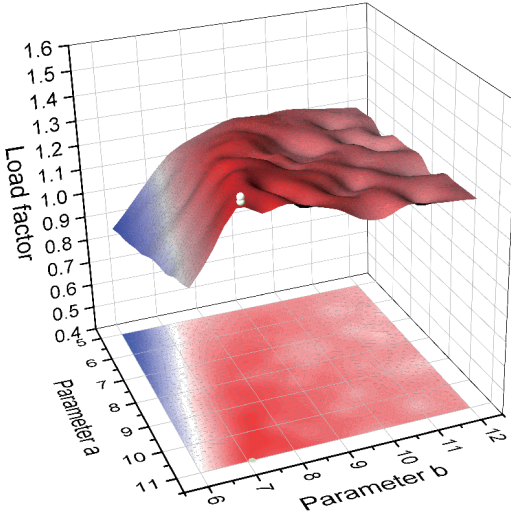

Generation 60

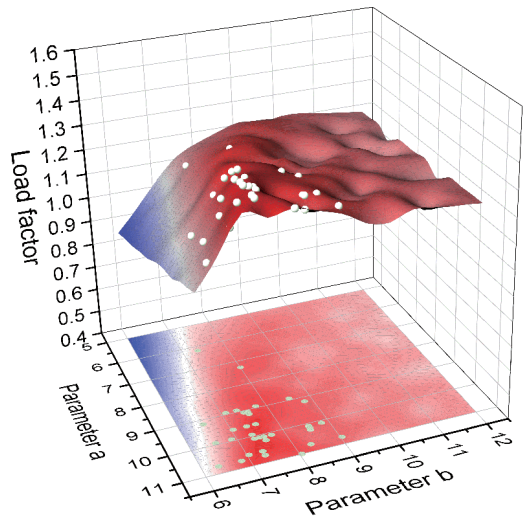

Generation 5

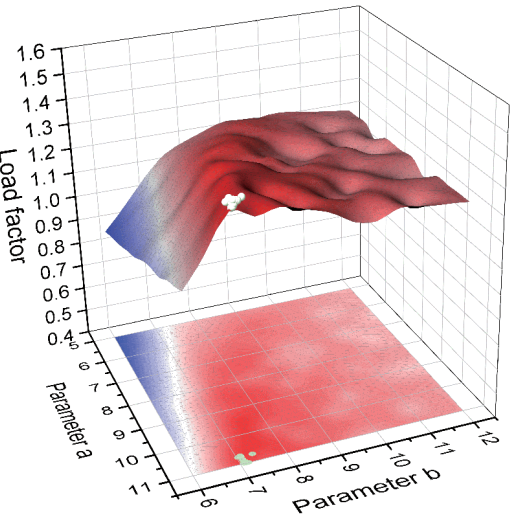

Generation 15

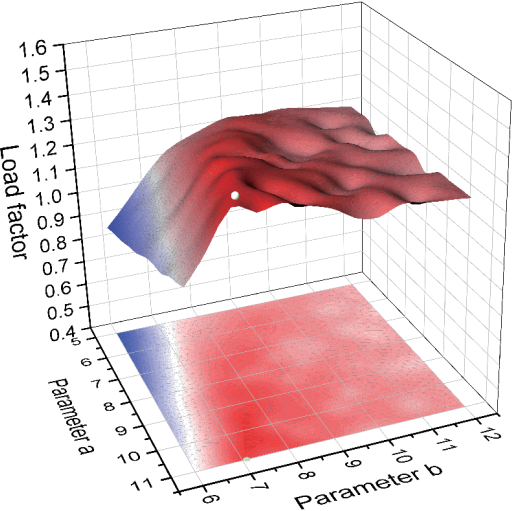

Generation 71 \& Final generation

Shakedown load factor

Figure 7 Population distribution in selected generations of optimization for maximum shakedown load 


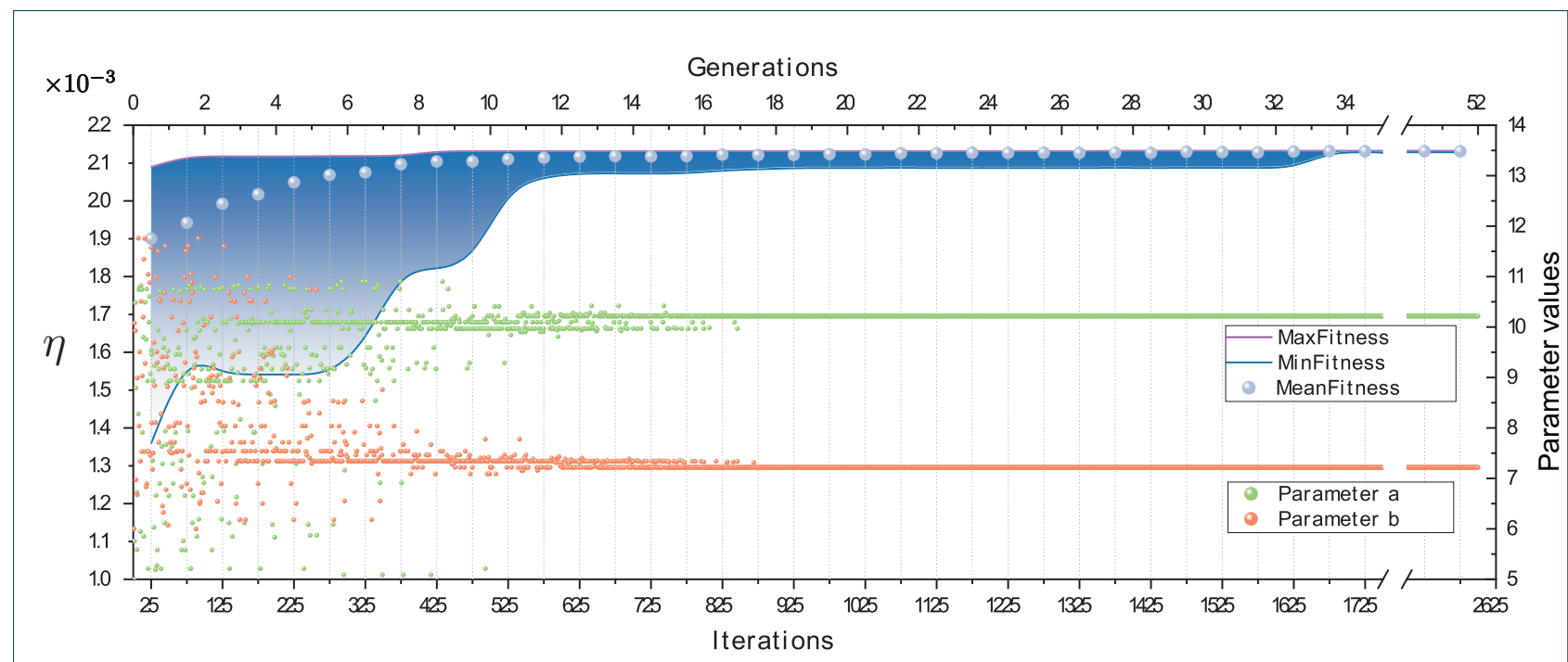

Figure 8 Convergence process of $\eta$ and its associated design parameters

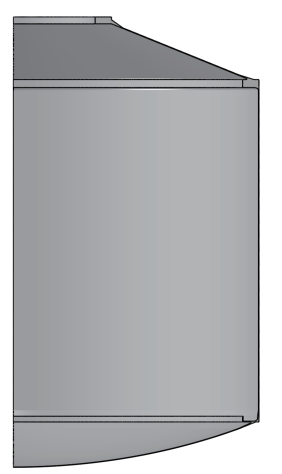

Undeformed structure

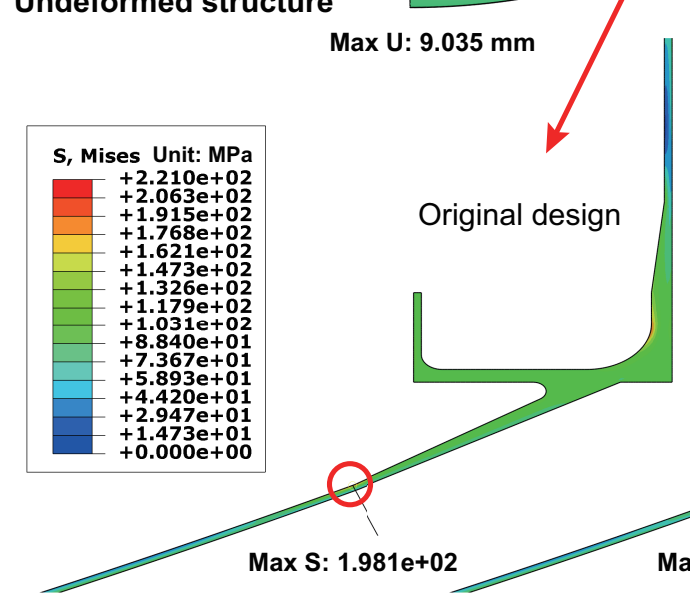

Max U: 8.544 mm

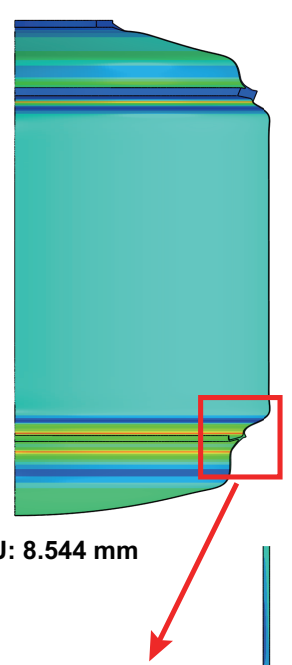

Optimal shakedown load design

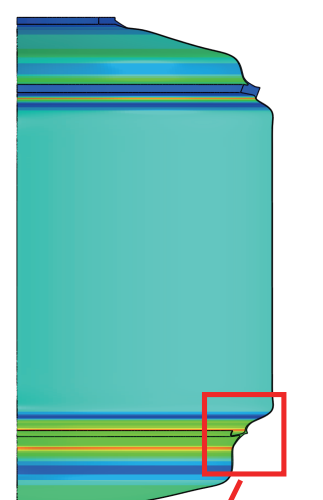

strength-to-weight efficiency design

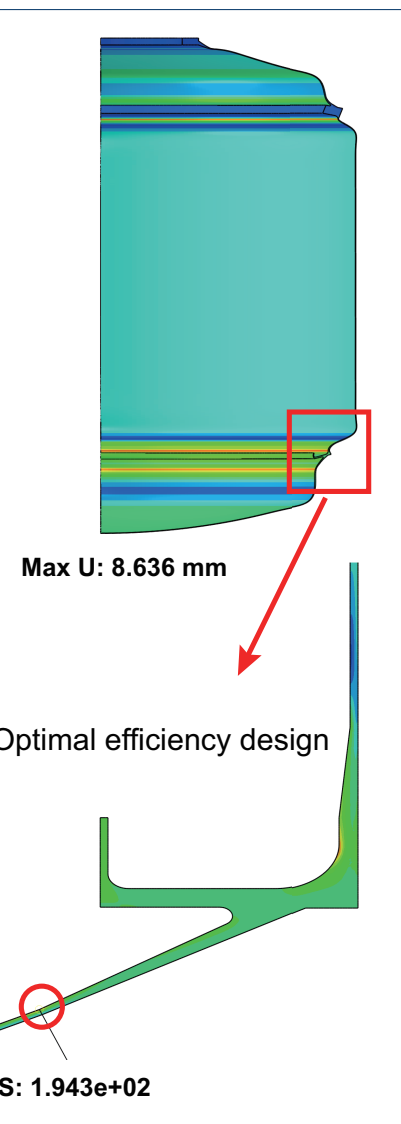

\section{Max S: $1.943 \mathrm{e}+02$}

Figure 9 Equivalent von Mises stress distribution on structure under original design, optimal shakedown load design and optimal 